\title{
TWO-DIMENSIONAL HELIOSEISMIC INVERSIONS
}

\section{J. SCHOU}

Institut for Fysik og Astronomi, Aarhus Universitet, Denmark, and High Altitude Observatory, NCAR *, Boulder, Colorado, USA

\section{J. CHRISTENSEN-DALSGAARD}

Institut for Fysik og Astronomi, Aarhus Universitet, Denmark

\section{J. THOMPSON}

Astronomy Unit, Queen Mary and Westfield College, London, England

\section{INTRODUCTION}

We present results of investigations of different inversion methods for inferring from helioseismic observations the rotation rate in the solar interior as a function of radius and latitude, using a mode set similar to that which is expected from the GONG network.

The rotation of a star perturbs the frequency $\omega_{n l m}$ of a normal mode by

$$
\Delta \omega_{n l m}=\omega_{n l m}-\omega_{n l 0}=\int_{0}^{R} \int_{0}^{\pi} K_{n l m}(r, \theta) \Omega(r, \theta) r d r d \theta,
$$

where $n, l$ and $m$ are the mode 'quantum' numbers, $\theta$ the colatitude, $\Omega$ the rotation rate and $K_{n l m}$ a known function. Until now the observations have not been in terms of individual $\Delta \omega_{n l m}$ 's; rather, coefficients $a_{i}(n, l)$ of a parametrization of the $m$-dependence of $\Delta \omega_{n l m}$ have been given. By representing the latitudinal dependence of the rotation rate in terms of an expansion in $\cos \theta$, an inversion of the $a_{i}$ can be carried out as a series of 1-dimensional inversions. Such inversions have been dubbed "1.5-dimensional". Our present implementation of a 1.5D procedure follows Schou et al. (1992), and uses $a_{1}, a_{3}$ and $a_{5}$.

By contrast a full 2-dimensional inversion uses all the individual $\Delta \omega_{n / m}$. Our implementation minimizes a discretized version of

$$
\sum_{i}\left(\frac{\int K_{i} \bar{\Omega} r d \theta d r-\Delta \omega_{i}}{\sigma_{i}}\right)^{2}+\mu \int\left[f(r, \theta)\left(\frac{\partial^{2} \bar{\Omega}}{\partial r^{2}}\right)^{2}+g(r, \theta)\left(\frac{\partial^{2} \bar{\Omega}}{\partial \theta^{2}}\right)^{2}\right] d \theta d r
$$

(Schou 1991; cf. Sekii 1990,1991$)^{\text {). Here }}{ }_{i}$ stands for the combination ${ }_{n i m}, \mu$ is a trade-off parameter, $\sigma_{i}$ are the errors on the observed splittings, $f$ and $g$ are functions of $r$ and $\theta$ that can be specified (here $f=1$ and $g=r^{-4}$ ), and $\bar{\Omega}$ is the rotation rate inferred from the inversion.

\footnotetext{
* NCAR is sponsored by the National Science Foundation.
} 


\section{RESULTS}

Figure 1 shows the inferred rotation rate $\bar{\Omega}\left(r_{0}, \theta_{0}\right)$ obtained with the two methods from splittings computed for an assumed "true" rotation rate. We have used a mode set comprising all modes with $l=0-200$ and $\nu=1-4 \mathrm{mHz}$, which is roughly what is expected from the GONG network. The assumed errors were estimated from the mode linewidths, and should represent the errors in real data. The trade-off parameters have been adjusted to obtain the same error for both methods. Superficially both methods reproduce the original rotation rate with reasonable precision. Error estimates show that with a realistic dataset one should be able to determine the rotation rate with considerable precision: for example, the standard error in $\bar{\Omega}$ is approximately $1 \mathrm{nHz}$ at $r_{0}=0.5 R$ on the equator.
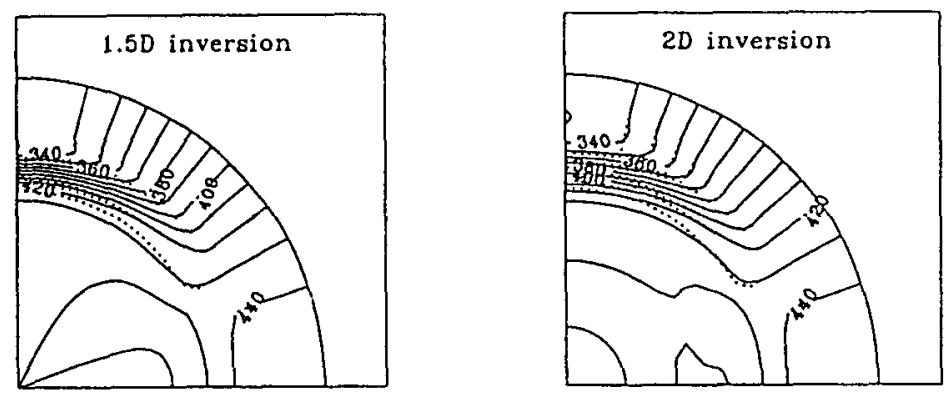

Fig. 1. The inferred rotation rate from $1.5 \mathrm{D}$ and $2 \mathrm{D}$ inversions, in a single quadrant with the North pole towards the top. Contours are labelled by the rotation rate, in $\mathrm{nHz}$. Dotted contours show the "true" rotation rate.

For both methods averaging kernels $\mathcal{K}\left(r_{0}, \theta_{0}, r, \theta\right)$ exist which relate the inferred and true rotation rates (Schou et al. 1992):

$$
\bar{\Omega}\left(r_{0}, \theta_{0}\right)=\int_{0}^{R} \int_{0}^{\pi} \mathcal{K}\left(r_{0}, \theta_{0}, r, \theta\right) \Omega(r, \theta) r d r d \theta .
$$

As illustrated in Figures 2 and 3 these provide a more sensitive impression of the properties of the inversions. It is evident that the $2 \mathrm{D}$ inversion has better latitudinal resolution: the reason is the $2 \mathrm{D}$ method uses information that is effectively thrown away when expanding in a-coefficients. Note that the improvement in resolution is achieved with the same errors in the data, and similar errors in the inferred $\bar{\Omega}$, as for the $1.5 \mathrm{D}$ case.

\section{REFERENCES}

Schou, J., 1991, In Lecture Notes in Physics vol. 388, 93 - 96, eds D. O. Gough \& J. Toomre, Springer, Berlin.

Schou, J., Christensen-Dalsgaard, J., \& Thompson, M. J., 1992, Ap. J., 385, L59- L62. 
Sekii, T., 1990. In Lecture Notes in Physics, vol, 367, 337 - 340, eds Osaki, Y. \& Shibahashi, H., Springer, Berlin.

Sekii, T., 1991. Publ. Astron. Soc. Japan, 43, $381-411$.

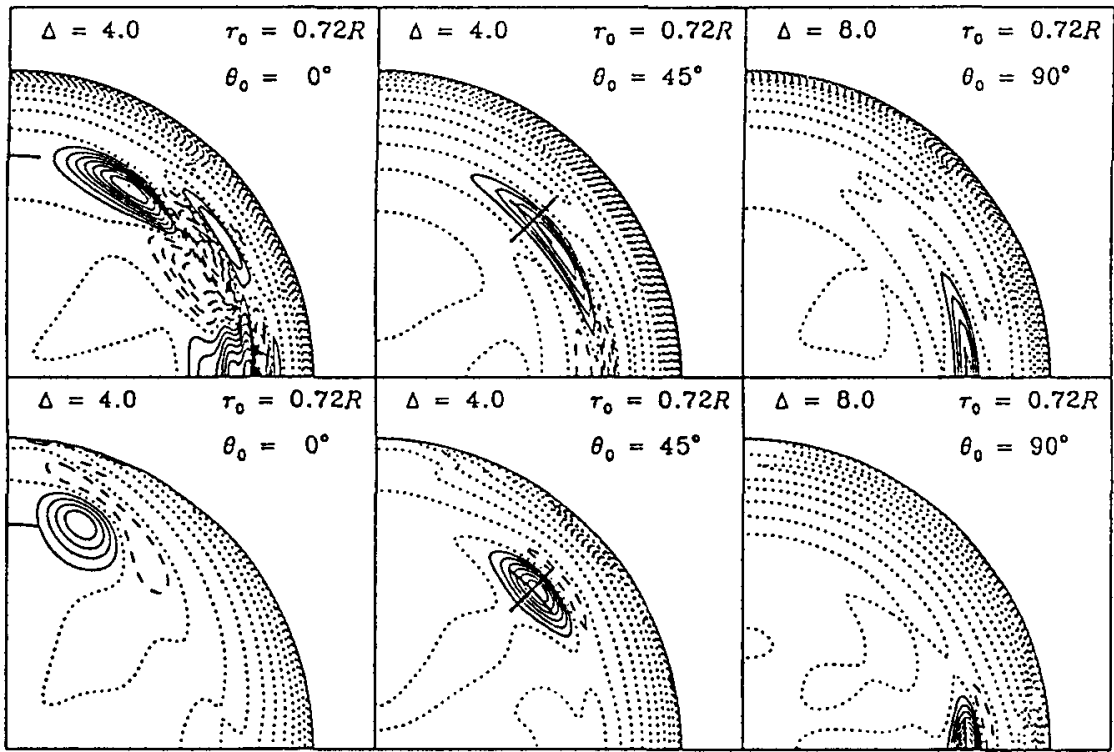

Fig. 2. Averaging kernels $\mathcal{K}\left(r_{0}, \theta_{0}, r, \theta\right)$ for the $1.5 \mathrm{D}$ (top) and $2 \mathrm{D}$ (bottom) inversions. Solid, dotted and dashed lines show contours for positive, zero and negative values, respectively. Heavy crosses show the location of the target position $\left(r_{0}, \theta_{0}\right) ; \Delta$ is the contour spacing.

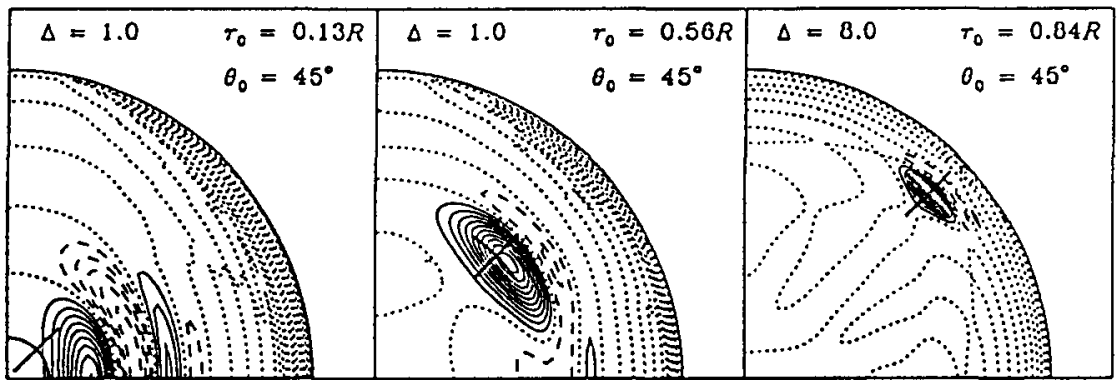

Fig. 3. Averaging kernels $\mathcal{K}\left(r_{0}, \theta_{0}, r, \theta\right)$ for the $2 \mathrm{D}$ inversion, at $\theta_{0}=45^{\circ}$, for three different target radii. See the caption to Figure 2 for explanations. 